\title{
Charismatic Leadership Studies- Past, Present and Future: Theorizing the Potential of Charismatic Rhetoric Research in Doctor of Education Programs
}

\author{
Simone Elias \\ Abilene Christian University \\ United States \\ E-mail: sse15a@acu.edu \\ Michael O Akintayo (Corresponding author) \\ Abilene Christian University \\ United States \\ E-mail: bikko63@aol.com
}

Received: April 4, 2018 Accepted: June 5, 2018 Published: December 23, 2018

doi:10.5296/ijssr.v7i1.12959 URL: http://dx.doi.org/10.5296/ijssr.v7i1.12959

\begin{abstract}
This theoretical review examines selected literature concerning charismatic leadership theory. It starts with an introduction of Max Weber's (1947) ground breaking application of the term "charisma" in the field of Social Sciences. Subsequently, it presents a research table, chronologically organized reflecting significant articles that highlight traits and historical developments of the charismatic leadership theory. Next, beginning with Weber's (1947) seminal article, this literature review discusses how the construct of charismatic leadership has advanced over the years. The examination largely concludes with a discussion of the current state of charismatic leadership, and a call for future studies to address charismatic rhetoric research in Doctor of education programs.
\end{abstract}

Keywords: charismatic leadership, rhetoric, Weber, leadership, charisma, education 


\section{MlMacrothink}

\section{Charismatic Leadership}

Weber's (1947) ground breaking application of the term "charisma" in the field of Social Sciences opened the doors for a scholarly discussion that seems to still be a work in progress in the 21 st century scholarly literature. Weber defined the term "charisma" as, "a certain quality of an individual personality by virtue of which he is considered extraordinary and treated as endowed with supernatural, superhuman or at least specifically exceptional powers or qualities" (p. 358). Weber emphasized that such qualities were, "not accessible to the ordinary person, but are regarded as of divine origin or as exemplary and on the basis of them the individual concerned is treated as a leader" (p. 359).

Despite the fact that Conger and Kanungo (1994) argued that not enough empirical research was available to support Weber's theory, the same authors, years later, acknowledged Weber's notion of transcendence and exceptionality stating that a, "leader's charisma could be transformed into routines and other institutional vehicles that in essence, 'lived on beyond the leader" (Conger \& Kanungo, 1998, p. 27).

In spite of Weber's (1947) significant contributions to the field of leadership, some scholars, such as Conger and Kanungo (1987), raised thoughtful concerns about Weber's (1947) charismatic theory, and offered constructive critiques and advancements. Other scholars, such as Beyer (1999), suggested that a departure from Weber's original construct could, "blur the distinctiveness of the charismatic form of leadership by ignoring or downplaying integral aspects of charisma, especially the precipitating crisis, the radical vision, and subsequent systemic change" (p. 307). Overall the literature pointed out that Weber's (1947) seminal work certainly pioneered and contributed to a series of studies and scholarly discussions in the field of leadership, as it is highlighted chronologically on research table 1.

Table 1. Chronology of Leadership Theoretical Frameworks

\begin{tabular}{|c|c|c|c|c|}
\hline $\begin{array}{c}\text { Article/ Book } \\
\text { Title }\end{array}$ & Date/ Journal & $\begin{array}{l}\text { Author(s) / } \\
\text { Discipline }\end{array}$ & Synopsis & Contributions \\
\hline $\begin{array}{l}\text { The theory of } \\
\text { social and } \\
\text { economic } \\
\text { organization }\end{array}$ & 1947/ Free Press & $\begin{array}{l}\text { Weber, M. } \\
\text { Sociologist and } \\
\text { Political Economist }\end{array}$ & $\begin{array}{l}\text { - Defines the } \\
\text { characteristics of } \\
\text { "Charisma," the } \\
\text { "routinization" of } \\
\text { charisma and } \\
\text { evaluates "the } \\
\text { different types of } \\
\text { authority } \\
\text { "rational"; } \\
\text { "traditional" and } \\
\text { " charismatic" }\end{array}$ & $\begin{array}{l}\text { Seminal article } \\
\text { - Religious term } \\
\text { "charisma" } \\
\text { applied to social } \\
\text { science (Weber, } \\
\text { 1947, p. 298). } \\
\text { - Transforms } \\
\text { charisma through } \\
\text { "routinization" } \\
\text { (Weber, 1947, p. } \\
\text { 329). }\end{array}$ \\
\hline
\end{tabular}




\begin{tabular}{|c|c|c|c|c|}
\hline & & & $\begin{array}{l}\text { (Weber, 1947, p. } \\
\text { 328) } \\
\text { - Defines Charisma } \\
\text { (Weber 1947, p. } \\
\text { 358-359). } \\
\text { - "What is alone } \\
\text { important is how } \\
\text { the individual is } \\
\text { actually regarded } \\
\text { by those subject to } \\
\text { charismatic } \\
\text { authority, but his } \\
\text { 'followers' or } \\
\text { 'disciples' "(Weber, } \\
\text { 1947, p. 359). }\end{array}$ & $\begin{array}{l}\text { - Charisma is only } \\
\text { awakened or } \\
\text { tested, NOT } \\
\text { learned or taught } \\
\text { (Weber, 1947,p. } \\
\text { 367). } \\
\text { Charismatic } \\
\text { authority will } \\
\text { disappear if a } \\
\text { leader fails to } \\
\text { benefit followers } \\
\text { (Weber, 1947, p. } \\
\text { 360). } \\
\text { Charisma "may } \\
\text { involve a } \\
\text { subjective or } \\
\text { internal } \\
\text { reorientation born } \\
\text { out of suffering, } \\
\text { conflicts, or } \\
\text { enthusiasm" } \\
\text { (Weber, 1947, p. } \\
\text { 363). }\end{array}$ \\
\hline $\begin{array}{l}\text { 1) Toward a } \\
\text { behavioral theory } \\
\text { of charismatic } \\
\text { leadership in } \\
\text { organizational } \\
\text { settings }\end{array}$ & $\begin{array}{l}\text { 1987/ The Academy } \\
\text { of Management } \\
\text { Review }\end{array}$ & $\begin{array}{l}\text { Conger, J. A., \& } \\
\text { DBA. Harvard Business } \\
\text { School; Senior Research } \\
\text { Scientist, University of } \\
\text { Southern California } \\
\text { Kanungo, R. N. } \\
\text { Ph.D. McGill University, } \\
\text { current member of } \\
\text { Faculty of Management at } \\
\text { McGill }\end{array}$ & $\begin{array}{l}\text { - Theoretical paper } \\
\text { argued } \\
\text { Charismatic } \\
\text { Leadership has } \\
\text { been ignored by } \\
\text { organizational } \\
\text { theorists (Conger } \\
\text { \& Kanungo, 1987, } \\
\text { p. 637). } \\
\text { - Proposed “A } \\
\text { Behavioral } \\
\text { Framework for } \\
\text { Studying } \\
\text { Charisma” }\end{array}$ & $\begin{array}{l}\text { - Pointed out that } \\
\text { Weber (1947) } \\
\text { "ascribed a } \\
\text { revolutionary and } \\
\text { counter normative } \\
\text { quality to } \\
\text { charismatic } \\
\text { authority" but } \\
\text { lacked } \\
\text { "specificity" (p. } \\
\text { 638). } \\
\text { The authors } \\
\text { concurred with } \\
\text { Weber (1947) that }\end{array}$ \\
\hline
\end{tabular}




\begin{tabular}{|c|c|c|c|c|}
\hline & & & $\begin{array}{l}\text { (Conger \& } \\
\text { Kanungo, 1987, p. } \\
639 \text { ). }\end{array}$ & $\begin{array}{l}\text { charismatic } \\
\text { authority is } \\
\text { "unstable and } \\
\text { transitory" (p. } \\
\text { 644). } \\
\text { - Advanced } \\
\text { studies by the } \\
\text { "idea that } \\
\text { charisma is an } \\
\text { attritional } \\
\text { phenomenon" (p. } \\
\text { 639). } \\
\text { - The model } \\
\text { advanced Weber's } \\
\text { (1947) concept of } \\
\text { Charisma, and } \\
\text { sought to } \\
\text { "demystify" the } \\
\text { term (p. 645). }\end{array}$ \\
\hline $\begin{array}{l}\text { 2) A laboratory } \\
\text { study of } \\
\text { charismatic } \\
\text { leadership }\end{array}$ & $\begin{array}{l}\text { 1989/ Organizational } \\
\text { Behavior and Human } \\
\text { Decision Processes }\end{array}$ & $\begin{array}{l}\text { Howell, J. P., \& } \\
\text { Ph.D. University of } \\
\text { California, Irvine; } \\
\text { Professor Emeritus } \\
\text { Department of } \\
\text { Management New Mexico } \\
\text { State University } \\
\text { Peter J Frost } \\
\text { Ph.D. University } \\
\text { Minnesota Former Chair } \\
\text { and } \\
\text { Professor University of } \\
\text { British Columbia }\end{array}$ & $\begin{array}{l}\text { - Empirical Study } \\
\text { - The authors } \\
\text { examined three } \\
\text { leadership styles, } \\
\text { and two levels of } \\
\text { group productivity } \\
\text { norms on } \\
\text { individual's } \\
\text { adjustment to and } \\
\text { performance on an } \\
\text { ambiguous task } \\
\text { (Howell \& Frost, } \\
\text { 1989, p.244) } \\
\text { University of } \\
\text { British Columbia } \\
\text { completed "a task } \\
\text { under the direction } \\
\text { of either a }\end{array}$ & $\begin{array}{l}\text { - Advanced the } \\
\text { theory of } \\
\text { Charismatic } \\
\text { Leadership and } \\
\text { noted that } \\
\text { individuals "can } \\
\text { be trained to } \\
\text { exhibit } \\
\text { charismatic } \\
\text { behavior" Howell } \\
\text { \& Frost, 1989, p. } \\
\text { 645). } \\
\text { Proposed several } \\
\text { questions for } \\
\text { further } \\
\text { advancement of } \\
\text { charismatic } \\
\text { leadership related }\end{array}$ \\
\hline
\end{tabular}




\begin{tabular}{|c|c|c|c|c|}
\hline & & & $\begin{array}{l}\text { charismatic, } \\
\text { structuring, or } \\
\text { considerate leader" } \\
\text { (Howell \& Frost, } \\
\text { p. } 243 \text { ). }\end{array}$ & $\begin{array}{l}\text { to "personality } \\
\text { traits" and } \\
\text { "situational } \\
\text { determinants of } \\
\text { charismatic } \\
\text { leadership" } \\
\text { (Howell \& Frost, } \\
\text { p. 266). }\end{array}$ \\
\hline $\begin{array}{l}\text { 3)The } \\
\text { motivational } \\
\text { effects } \\
\text { charismatic } \\
\text { leadership:a } \\
\text { self-concept } \\
\text { based theory. }\end{array}$ & $\begin{array}{l}\text { 1993/Organization } \\
\text { Science }\end{array}$ & $\begin{array}{l}\text { Shamir, B., } \\
\text { Ph.D. Social Psychology } \\
\text { from London School of } \\
\text { Economics and Political } \\
\text { Science, Dean at Hebrew } \\
\text { University of Jerusalem } \\
\text { House, R. J., \& } \\
\text { Ph.D. Management Ohio } \\
\text { State University } \\
\text { Former professor } \\
\text { Wharton School, } \\
\text { Department of } \\
\text { Management, University } \\
\text { of Pennsylvania } \\
\text { Arthur, M. B. } \\
\text { Ph.D., MBA, Cranfield } \\
\text { School of } \\
\text { Management, England. } \\
\text { Professor School of } \\
\text { Management, Suffolk } \\
\text { University }\end{array}$ & 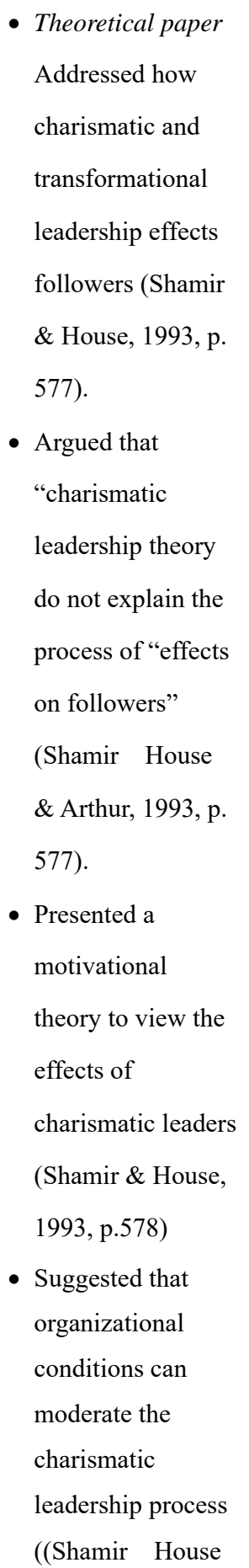 & $\begin{array}{l}\text { - Advanced theory } \\
\text { by "a theoretical } \\
\text { extension of } \\
\text { current theories of } \\
\text { charismatic and } \\
\text { transformational } \\
\text { leadership" } \\
\text { (Shamir \& House } \\
\text { \& Arthur, 1993, p. } \\
\text { 590). } \\
\text { Called for further } \\
\text { research and } \\
\text { empirical tests of } \\
\text { the “theoretical } \\
\text { extension" } \\
\text { ((Shamir House } \\
\text { \& Arthur, p. 591). }\end{array}$ \\
\hline
\end{tabular}




\begin{tabular}{|c|c|c|c|c|}
\hline & & & \& Arthur,p. 591). & \\
\hline $\begin{array}{l}\text { 4) Charismatic } \\
\text { leadership in } \\
\text { organizations: } \\
\text { perceived } \\
\text { behavioral } \\
\text { attributes and } \\
\text { their } \\
\text { measurement. }\end{array}$ & $\begin{array}{l}\text { 1994/ Journal of } \\
\text { Organizational } \\
\text { Behavior }\end{array}$ & $\begin{array}{l}\text { Conger, J. A., \& } \\
\text { DBA. Harvard } \\
\text { Business School, } \\
\text { Senior Research } \\
\text { Scientist, University of } \\
\text { Southern California, } \\
\text { Kanungo, R. } \\
\text { N. } \\
\text { Ph.D. McGill } \\
\text { University, Professor } \\
\text { of Management at } \\
\text { McGill University }\end{array}$ & 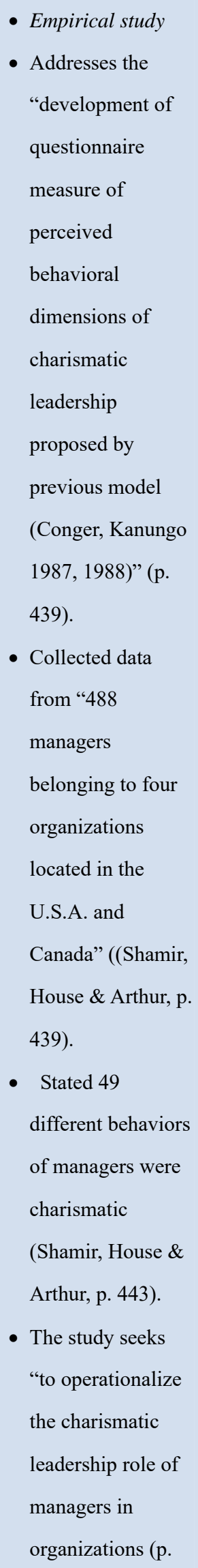 & $\begin{array}{l}\text { - Argued that } \\
\text { not enough empirical } \\
\text { research has been } \\
\text { done concerning } \\
\text { Weber's (1947) } \\
\text { theory (p. 440). } \\
\text { •Conger-Kanungo } \\
\text { (1994), examined } \\
\text { Scale of Charismatic } \\
\text { Leadership" (C-K } \\
\text { Scale) (p. } 443 \text { ). } \\
\text { - Suggested: the } \\
\text { "need for further } \\
\text { studies to vigorously } \\
\text { establish criterion } \\
\text { validity" and "to } \\
\text { explore empirically } \\
\text { the links between the } \\
\text { individual behavioral } \\
\text { dimensions and } \\
\text { specific follower } \\
\text { outcomes" (p. } 450 \text { ). }\end{array}$ \\
\hline
\end{tabular}




\begin{tabular}{|c|c|c|c|c|}
\hline & & & 443). & \\
\hline $\begin{array}{l}\text { 5) An evaluation } \\
\text { of conceptual } \\
\text { weaknesses in } \\
\text { transformational } \\
\text { and charismatic } \\
\text { leadership } \\
\text { theories }\end{array}$ & $\begin{array}{l}\text { 1999/ The Leadership } \\
\text { Quarterly }\end{array}$ & $\begin{array}{l}\text { Yukl, G. } \\
\text { Ph.D. } \\
\text { Industrial-Organizational } \\
\text { Psychology University of } \\
\text { California at } \\
\text { Berkeley } \\
\text { Professor of Management } \\
\text { and Psychology State } \\
\text { University of NY, Albany }\end{array}$ & $\begin{array}{l}\text { Theoretical paper } \\
\text { Suggests, "most of } \\
\text { the theories have } \\
\text { conceptual } \\
\text { weaknesses" } \\
\text { which limits the } \\
\text { successful } \\
\text { explanation of } \\
\text { leadership } \\
\text { phenomenon } \\
\text { (Yukl, 1999, p. } \\
\text { 285). } \\
\text { Discusses how } \\
\text { earlier theories } \\
\text { present weakness } \\
\text { (Yukl, 1999). } \\
\text { Discusses issue of } \\
\text { compatibility } \\
\text { between } \\
\text { transformational } \\
\text { and charismatic } \\
\text { leadership (Yukl, } \\
\text { 1999). }\end{array}$ & $\begin{array}{l}\text { - The author } \\
\text { discusses how } \\
\text { Weber's (1947) } \\
\text { theory advanced } \\
\text { (p. 297). } \\
\text { - Argues that "the } \\
\text { potential for using } \\
\text { charismatic } \\
\text { leadership to } \\
\text { improve } \\
\text { organizations } \\
\text { seems limited, and } \\
\text { it may be } \\
\text { warranted only in } \\
\text { special situations" } \\
\text { (Yukl, 1999, p. } \\
\text { 301). } \\
\text { - Advances the } \\
\text { theory of } \\
\text { Charismatic } \\
\text { Leadership by } \\
\text { stating that “little } \\
\text { research on } \\
\text { underlying } \\
\text { influence } \\
\text { processes" has conducted } \\
\text { (Yuk1, }\end{array}$ \\
\hline $\begin{array}{l}\text { 6) Taming and } \\
\text { promoting } \\
\text { charisma. }\end{array}$ & $\begin{array}{l}\text { 1999/ The Leadership } \\
\text { Quarterly }\end{array}$ & $\begin{array}{l}\text { Beyer, J. M. } \\
\text { Ph.D. Cornell; } \\
\text { Former Professor of } \\
\text { management at The } \\
\text { University of Texas at } \\
\text { Austin, distinguished } \\
\text { scholar. }\end{array}$ & $\begin{array}{l}\text { - Theoretical paper } \\
\text { Suggests that a } \\
\text { "sociological" } \\
\text { evaluation of the } \\
\text { literature on } \\
\text { charisma uncovers } \\
\text { "how theories and } \\
\text { ways of }\end{array}$ & $\begin{array}{l}\text { - States, } \\
\text { "neo-charismatic } \\
\text { and } \\
\text { transformational } \\
\text { leadership } \\
\text { paradigms have } \\
\text { tamed the original } \\
\text { conception of } \\
\text { charisma }\end{array}$ \\
\hline
\end{tabular}




\begin{tabular}{|c|c|c|c|c|}
\hline & & & $\begin{array}{l}\text { operationalizing } \\
\text { charisma depart } \\
\text { from Weber's } \\
\text { original } \\
\text { conception" p. } \\
\text { (Beyer, 1999, p. } \\
\text { 307). } \\
\text { Contends that } \\
\text { perhaps Charisma } \\
\text { “is more common } \\
\text { and less } \\
\text { extraordinary" } \\
\text { (Beyer, 1999, p. } \\
\text { 316). } \\
\text { The author } \\
\text { defines “charisma } \\
\text { as a social process } \\
\text { and emergent } \\
\text { social structure } \\
\text { that encompasses } \\
\text { more than the } \\
\text { leadership } \\
\text { process" (Beyer, } \\
\text { (B26). }\end{array}$ & $\begin{array}{l}\text { advanced by } \\
\text { Weber (1947) and, } \\
\text { in the process, } \\
\text { diluted its } \\
\text { richness and } \\
\text { distinctiveness" } \\
\text { (Beyer, 1999, p. } \\
\text { 308). } \\
\text { Argues the issue } \\
\text { with theorizing } \\
\text { che study } \\
\text { leadership"(Yukl, } \\
\text { 1999, p. 325). } \\
\text { property of a } \\
\text { person, a } \\
\text { theory through a } \\
\text { sociological } \\
\text { situation } \\
\text { (Yukl,1999, p. } \\
\text { approach a } \\
\text { explanation } \\
\text { Weber's (1947) } \\
\text { definition of } \\
\text { charisma (p. 313). } \\
\text { - }\end{array}$ \\
\hline $\begin{array}{l}\text { 7) Charismatic } \\
\text { and } \\
\text { transformational } \\
\text { leadership in } \\
\text { organizations: an } \\
\text { insider's } \\
\text { perspective on } \\
\text { these developing } \\
\text { streams of } \\
\text { research. }\end{array}$ & $\begin{array}{l}\text { 1999/ The Leadership } \\
\text { Quarterly }\end{array}$ & $\begin{array}{l}\text { Conger, J. A. } 1999 \\
\text { DBA - Harvard Business } \\
\text { School, Professor of } \\
\text { Leadership Studies, } \\
\text { Claremont McKenna } \\
\text { College }\end{array}$ & $\begin{array}{l}\text { - Theoretical paper } \\
\text { "Examines the } \\
\text { "evolution" of } \\
\text { Transformational } \\
\text { leadership in } \\
\text { organizations. } \\
\text { - Looks at } \\
\text { "background } \\
\text { forces driving the }\end{array}$ & $\begin{array}{l}\text { - Builds upon } \\
\text { Weber's } \\
\text { conception of } \\
\text { charisma } \\
\text { - Advances the } \\
\text { theory by arguing } \\
\text { "Weber's notion } \\
\text { that charisma } \\
\text { must ultimately } \\
\text { institutionalize } \\
\text { itself may no } \\
\text { longer be }\end{array}$ \\
\hline
\end{tabular}




\begin{tabular}{|c|c|c|c|c|}
\hline & & & $\begin{array}{l}\text { growth of interest" } \\
\text { in transformational } \\
\text { and Charismatic } \\
\text { leadership } \\
\text { (Conger, 1999, p. } \\
\text { 147). }\end{array}$ & $\begin{array}{l}\text { accurate", due to } \\
\text { globalization and } \\
\text { new era (Conger, } \\
\text { 1999, p. 170). }\end{array}$ \\
\hline $\begin{array}{l}\text { 8) Charismatic } \\
\text { leadership: } \\
\text { strategies for } \\
\text { effecting social } \\
\text { change. }\end{array}$ & $\begin{array}{l}\text { 1999/ The Leadership } \\
\text { Quarterly }\end{array}$ & $\begin{array}{l}\text { Fiol, C., } \\
\text { Ph.D., University of } \\
\text { Illinois; } \\
\text { Professor of } \\
\text { Management, University } \\
\text { of Colorado at Denver } \\
\text { Harris, D., \& } \\
\text { Ph.D., Management New } \\
\text { York University; former } \\
\text { professor Fairleigh } \\
\text { Dickinson University; } \\
\text { House, } \quad \text { R. } \\
\text { (1999), } \\
\text { Ph.D. Management Ohio } \\
\text { State University } \\
\text { Former professor } \\
\text { Wharton School, } \\
\text { Department of } \\
\text { Management, University } \\
\text { of Pennsylvania }\end{array}$ & $\begin{array}{l}\text { - Empirical study } \\
\text { - Developed a } \\
\text { chrocess model" of } \\
\text { leadership utilizing } \\
\text { theories of "social } \\
\text { meaning" (Fiol, } \\
\text { Harris, \&House, } \\
\text { 1999, p. } 449 \text { ). } \\
\text { The model } \\
\text { leaders employ } \\
\text { consistent } \\
\text { clarifies "why and } \\
\text { thricate, } \\
\text { how the }\end{array}$ & $\begin{array}{l}\text { Advanced the } \\
\text { theory by arguing } \\
\text { that while Conger } \\
\text { \& Kanungo, } 1987 \\
\text { and Weber, } 1947 \\
\text { suggest that } \\
\text { charismatic } \\
\text { leaders can effect } \\
\text { change the } \\
\text { "redirect } \\
\text { attention" to } \\
\text { authors theoretical } \\
\text { theory of } \\
\text { feadership by } \\
\text { framework and } \\
\text { et al, p.470). } \\
\text { results show } \\
\text { charismatic } \\
\text { processicalo } \\
\text { leadership cannot } \\
\text { be represented by } \\
\text { a single }\end{array}$ \\
\hline
\end{tabular}




\begin{tabular}{|c|c|c|c|c|}
\hline & & & $\begin{array}{l}\text { moving, and } \\
\text { re-aligning the } \\
\text { norms of their } \\
\text { followers" (Fiol, } \\
\text { Harris, \& House, } \\
\text { p. } 450 \text { ). }\end{array}$ & \\
\hline $\begin{array}{l}\text { 9) The effects of } \\
\text { visionary and } \\
\text { crisis-responsive } \\
\text { charisma on } \\
\text { followers: an } \\
\text { experimental } \\
\text { examination of } \\
\text { two kinds of } \\
\text { charismatic } \\
\text { leadership. }\end{array}$ & $\begin{array}{l}\text { 1999/ The Leadership } \\
\text { Quarterly }\end{array}$ & $\begin{array}{l}\text { Hunt, J. G., } \\
\text { Ph.D. } \\
\text { University of Illinois; } \\
\text { Former Professor, } \\
\text { College of Business Texas } \\
\text { Tech University } \\
\text { Boal, K. B., \& } \\
\text { Ph.D. University of } \\
\text { Wisconsin-Madison; } \\
\text { Professor, College of } \\
\text { Business Texas Tech } \\
\text { University } \\
\text { Dodge, G. E. } \\
\text { Former Professor, } \\
\text { College of Business Texas } \\
\text { Tech University }\end{array}$ & 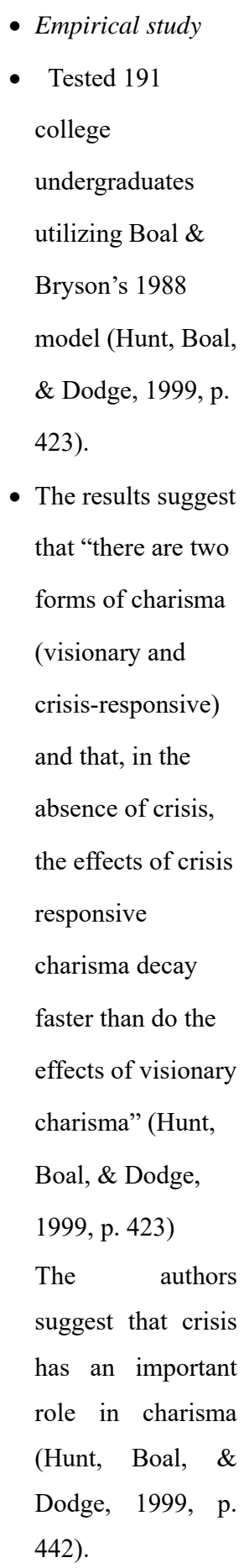 & $\begin{array}{l}\text { - Challenges } \\
\text { Weber's (1947) } \\
\text { definition of } \\
\text { leader as } \\
\text { "extraordinary" } \\
\text { (p. 443). } \\
\text { Provides support } \\
\text { for hypotheses of } \\
\text { the existence of } \\
\text { two kinds of } \\
\text { leadership; } \\
\text { "visionary" and } \\
\text { "crisis-responsive } \\
\text { ” (Hunt, Boal, \& } \\
\text { Dodge, 1999, p. } \\
\text { 425). } \\
\text { Advances the } \\
\text { theory by } \\
\text { suggesting, } \\
\text { "Crisis alone does } \\
\text { not create } \\
\text { charisma but } \\
\text { crisis and } \\
\text { response does" } \\
\text { (Hunt, Boal, \& } \\
\text { Dodge, 1999, p. } \\
\text { (H). }\end{array}$ \\
\hline $\begin{array}{l}\text { 10) Charismatic } \\
\text { leadership and }\end{array}$ & $\begin{array}{l}\text { 2000/ Journal of } \\
\text { Organizational }\end{array}$ & $\begin{array}{l}\text { Conger, J. A., } \\
\text { DBA - Harvard Business }\end{array}$ & - Empirical study & - Data/scale \\
\hline
\end{tabular}




\begin{tabular}{|c|c|c|c|c|}
\hline follower effects. & Behavior & $\begin{array}{l}\text { School, Professor of } \\
\text { Leadership Studies, } \\
\text { Claremont McKenna } \\
\text { College. } \\
\text { Kanungo, R. N., \& } \\
\text { Ph.D. McGill University, } \\
\text { Professor of Management } \\
\text { at McGill University. } \\
\text { Menon, S. T. } \\
\text { Ph.D. McGill University, } \\
\text { Professor of Management } \\
\text { at School of Business, } \\
\text { Clarkson University }\end{array}$ & 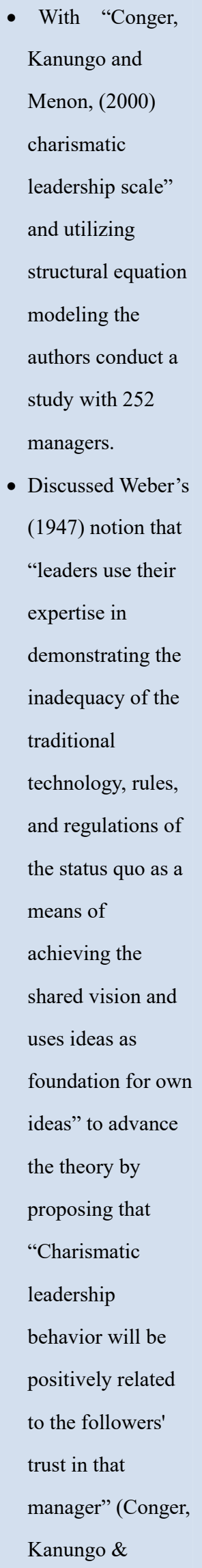 & 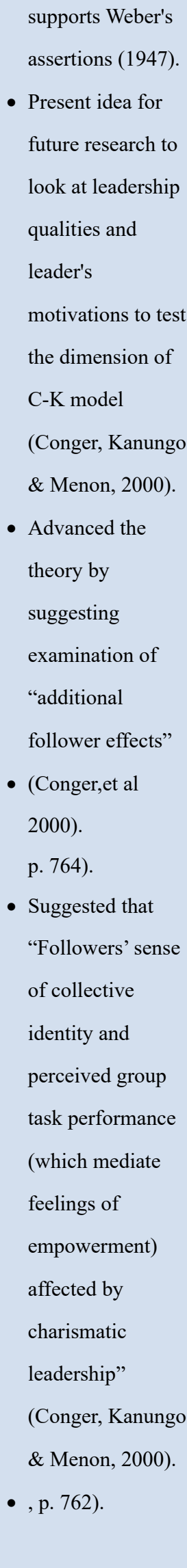 \\
\hline
\end{tabular}




\begin{tabular}{|c|c|c|c|c|}
\hline & & & $\begin{array}{l}\text { Menon, 2000). } \\
\text { - p.750). }\end{array}$ & \\
\hline $\begin{array}{l}\text { 11) The effects of } \\
\text { charismatic } \\
\text { leadership on } \\
\text { followers' } \\
\text { self-concept } \\
\text { accessibility. }\end{array}$ & $\begin{array}{l}\text { 2001/ Journal of } \\
\text { Applied Social } \\
\text { Psychology, }\end{array}$ & $\begin{array}{l}\text { Paul, J., } \\
\text { Ph.D. New Mexico State } \\
\text { University, Professor } \\
\text { University of Kansas } \\
\text { Medical Center } \\
\text { Costley, D. L., } \\
\text { Former professor } \\
\text { Department of } \\
\text { Management New Mexico } \\
\text { State University } \\
\text { Howell, J. P., \& Ph.D. } \\
\text { University of California, } \\
\text { Irvine; Professor } \\
\text { Emeritus Department of } \\
\text { Management New Mexico } \\
\text { State University } \\
\text { Dorfman, P. W. } \\
\text { Ph.D. University of } \\
\text { Maryland. Professor } \\
\text { Emeritus Department of } \\
\text { Management New Mexico } \\
\text { State University } \\
\text { Trafimow, D } \\
\text { Ph.D. Social } \\
\text { Psychology, University of } \\
\text { Illinois at } \\
\text { Urbana-Champaign, } \\
\text { Professor Department of } \\
\text { Management New Mexico } \\
\text { State University }\end{array}$ & 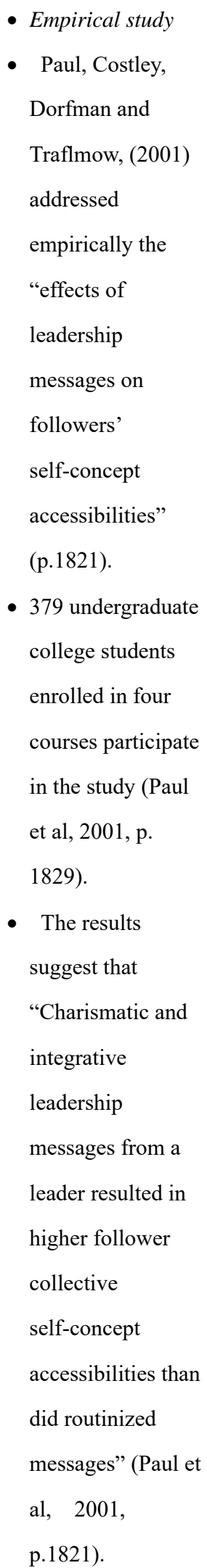 & 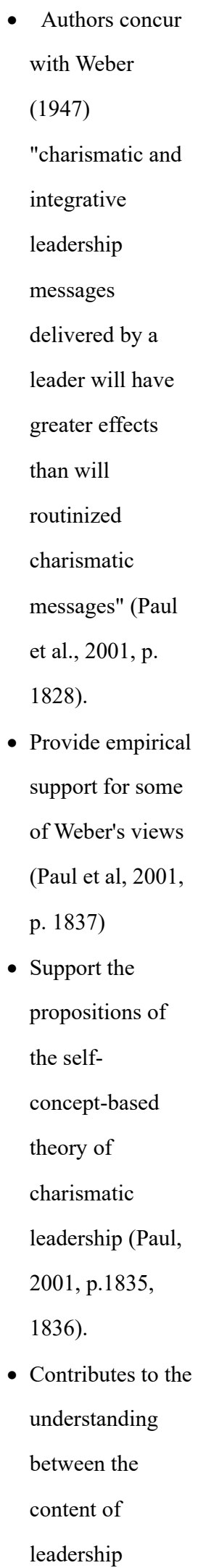 \\
\hline
\end{tabular}




\begin{tabular}{|c|c|c|c|c|}
\hline & & & $\begin{array}{l}\text { Notes the } \\
\text { importance of a } \\
\text { charismatic leader } \\
\text { (p.1821). } \\
\text { - Findings provide } \\
\text { "some empirical } \\
\text { support for the } \\
\text { views of Weber } \\
\text { (1947) and } \\
\text { Weierter (1997) } \\
\text { that a leader } \\
\text { remains important } \\
\text { to the effectiveness } \\
\text { of charismatic } \\
\text { leadership even } \\
\text { when charismatic } \\
\text { messages have } \\
\text { become } \\
\text { routinized" (p. } \\
\text { 1837) }\end{array}$ & $\begin{array}{l}\text { messages and the } \\
\text { communication of } \\
\text { leadership } \\
\text { messages (Paul et } \\
\text { al, p. 1837). }\end{array}$ \\
\hline $\begin{array}{l}\text { 12) An affective } \\
\text { events model of } \\
\text { charismatic } \\
\text { leadership } \\
\text { behavior: A } \\
\text { review, theoretical } \\
\text { integration, and } \\
\text { research agenda. }\end{array}$ & $\begin{array}{l}\text { 2009/ Journal of } \\
\text { Management }\end{array}$ & $\begin{array}{l}\text { Walter, F., \& } \\
\text { Ph.D. Business } \\
\text { St. Gallen University; } \\
\text { Switzerland, Professor } \\
\text { University of Groningen, } \\
\text { Netherlands. } \\
\text { Bruch, H. } \\
\text { Ph.D. Business } \\
\text { University of St. Gallen, } \\
\text { Switzerland; Professor } \\
\text { University of St. Gallen, } \\
\text { Switzerland; }\end{array}$ & $\begin{array}{l}\text { - Theoretical paper } \\
\text { - Reviews studies on } \\
\text { charismatic } \\
\text { leadership } \\
\text { behavior } \\
\text { emergence and } \\
\text { seeks a } \\
\text { "comprehensive } \\
\text { perspective by } \\
\text { integrating } \\
\text { previous work into } \\
\text { an overall } \\
\text { conceptual } \\
\text { framework" } \\
\text { (Walter \& Bruch, } \\
2009, \text { p. 1428). } \\
\text { The authors }\end{array}$ & $\begin{array}{l}\text { - Advances the } \\
\text { theory by } \\
\text { suggesting } \\
\text { scholars such } \\
\text { Conger (1999) } \\
\text { and Yukl (1999) } \\
\text { have "neglected } \\
\text { the origins of } \\
\text { charismatic } \\
\text { leadership } \\
\text { behavior" } \\
\text { Incorporated } \\
\text { "individual and } \\
\text { contextual } \\
\text { influencing } \\
\text { factors" (Conger, } \\
\text { 1999, p. 1438). }\end{array}$ \\
\hline
\end{tabular}




\begin{tabular}{|c|c|c|c|c|}
\hline & & & 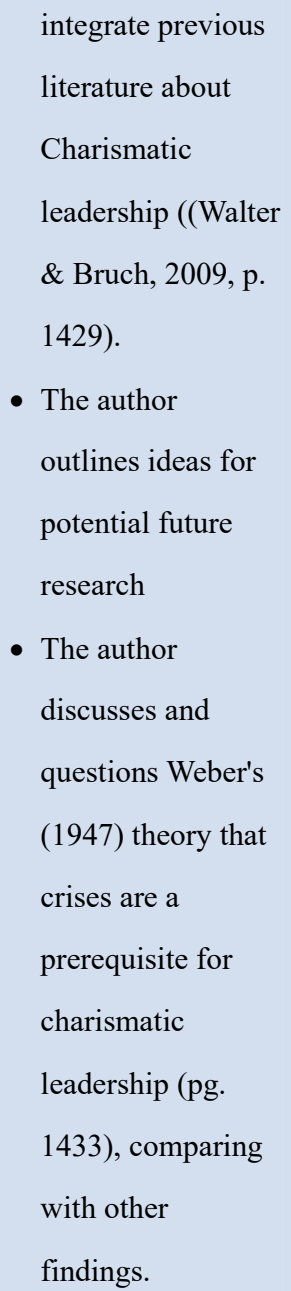 & $\begin{array}{l}\text { - Advances the } \\
\text { leadership Theory } \\
\text { by providing } \\
\text { "solid foundation } \\
\text { for further } \\
\text { scholarly efforts" } \\
\text { (p. 1429). } \\
\text { Promotes "a more } \\
\text { inclusive } \\
\text { depiction of } \\
\text { charismatic } \\
\text { leadership } \\
\text { behavior } \\
\text { emergence" } \\
\text { (Walter \& Bruch, } \\
\text { 2009, p. 1444). }\end{array}$ \\
\hline $\begin{array}{l}\text { 13) Measuring } \\
\text { transformational } \\
\text { and charismatic } \\
\text { leadership: why } \\
\text { isn't charisma } \\
\text { measured. }\end{array}$ & $\begin{array}{l}\text { 2010/Communication } \\
\text { Monographs }\end{array}$ & $\begin{array}{l}\text { Levine, K. J., } \\
\text { PhD, Michigan State; } \\
\text { Associate Professor } \\
\text { School of Communication } \\
\text { Studies at the University } \\
\text { of Tennessee } \\
\text { Muenchen, R. A., } \\
\text { M.A., Tennessee; Director } \\
\text { of the Statistical } \\
\text { Consulting Center at the } \\
\text { University of Tennessee } \\
\text { Brooks, A. } \quad \text { M. } \\
\text { PhD, Tennessee Assistant } \\
\text { Professor at Georgia } \\
\text { Southern University }\end{array}$ & $\begin{array}{l}\text { - Empirical study } \\
\text { - Conducted a study } \\
\text { with } 422 \\
\text { participants and } \\
\text { utilizes "text } \\
\text { analysis and } \\
\text { correlations" } \\
\text { (Levine, Mychen, } \\
\text { Brooks, p. 576). } \\
\text { Argued that the } \\
\text { measurement } \\
\text { scales utilized to } \\
\text { measure } \\
\text { Charismatic and } \\
\text { Transformational } \\
\text { theories "fail to }\end{array}$ & $\begin{array}{l}\text { A review of } \\
\text { studies about } \\
\text { Charisma, } \\
\text { including Weber } \\
\text { (1947); Conger } \\
\text { (1989) and other } \\
\text { important } \\
\text { scholars, unveiled } \\
\text { that verbs are } \\
\text { used more } \\
\text { frequently to } \\
\text { define charismatic } \\
\text { leadership. } \\
\text { skills "should be } \\
\text { evaluated when }\end{array}$ \\
\hline
\end{tabular}




\begin{tabular}{|c|c|c|c|c|}
\hline & & & $\begin{array}{l}\text { adequately address } \\
\text { and measure the } \\
\text { communication } \\
\text { behaviors that are } \\
\text { believed to be a } \\
\text { part of charisma" } \\
\text { (Walter \& Bruch, } \\
2009, p .576) \text {. } \\
\text { Conclude there is } \\
\text { no relation } \\
\text { between current } \\
\text { scales and aspects } \\
\text { of charismatic } \\
\text { behavior ((Walter } \\
\& \text { Bruch, 2009, } \\
\text { p.586). }\end{array}$ & $\begin{array}{l}\text { assessing a } \\
\text { charismatic } \\
\text { leader" (Walter \& } \\
\text { Bruch, 2009,p. } \\
\text { 580). } \\
\text { - Challenges } \\
\text { current scales, } \\
\text { argues that they } \\
\text { are not accurate } \\
\text { (p. 582). } \\
\text { Suggested the } \\
\text { creation of a } \\
\text { leadership } \\
\text { measurement } \\
\text { including } \\
\text { charismatic } \\
\text { communication } \\
\text { behaviors ((Walter } \\
\text { \& Bruch, 2009, p. } \\
589) .\end{array}$ \\
\hline $\begin{array}{l}\text { 14) Can charisma } \\
\text { be taught? Tests } \\
\text { of two } \\
\text { interventions. }\end{array}$ & $\begin{array}{l}\text { 2011/ Academy of } \\
\text { Management } \\
\text { Learning \& } \\
\text { Education }\end{array}$ & $\begin{array}{l}\text { Antonakis, J., } \\
\text { Professor University of } \\
\text { Lausanne } \\
\text { Fenley, M., \& } \\
\text { Professor University of } \\
\text { Lausanne } \\
\text { Liechti, S. } \\
\text { Professor University of } \\
\text { Lausanne }\end{array}$ & $\begin{array}{l}\text { - Empirical study } \\
\text { - Conducted studies } \\
\text { to evaluate if it is } \\
\text { possible to "teach } \\
\text { individuals to } \\
\text { behave more } \\
\text { charismatically, } \\
\text { and if changes in } \\
\text { charisma affect } \\
\text { leader outcomes" } \\
\text { (Anonakis, Fenep. } \\
374) . \\
\text { Studies utilized “a } \\
\text { mixed-design field } \\
\text { experiment" with } \\
\text { "34 middle-level” } \\
\text { participants } \\
\text { ((Walter \& Bruch, } \\
2009, \text { p. } 374) .\end{array}$ & $\begin{array}{l}\text { - Discuss the } \\
\text { charisma } \\
\text { phenomenon and } \\
\text { suggests } \\
\text { "Leadership is } \\
\text { learnable" } \\
\text { (Antonakis, } \\
\text { Fenley \& } \\
\text { Liechtip. 390). } \\
\text { Challenges } \\
\text { Weber's (1947) } \\
\text { suggestion of } \\
\text { "charisma as a gift } \\
\text { of the body and } \\
\text { spirit not } \\
\text { accessible to } \\
\text { everybody" (p. } \\
\text { 375). } \\
\text { Suggests that }\end{array}$ \\
\hline
\end{tabular}




\begin{tabular}{|c|c|c|c|c|}
\hline & & & $\begin{array}{l}\text { - Second study uses } \\
\text { "within-subjects } \\
\text { laboratory } \\
\text { experiment" with } \\
\text { “41 MBA } \\
\text { participants giving } \\
\text { a speech" ((Walter } \\
\text { \& Bruch, 2009, p. } \\
\text { 374). } \\
\text { The experiments } \\
\text { suggest "that } \\
\text { leaders appear } \\
\text { charismatic } \\
\text { because they use a } \\
\text { wide array of } \\
\text { verbal and } \\
\text { nonverbal } \\
\text { Charismatic } \\
\text { Leadership } \\
\text { Tactics" (Walter \& } \\
\text { Bruch, p. 390). }\end{array}$ & $\begin{array}{l}\text { future research } \\
\text { "focus on } \\
\text { uncovering which } \\
\text { learning processes } \\
\text { are most relevant } \\
\text { and how they can } \\
\text { be managed and } \\
\text { accelerated" } \\
\text { (Walter \& Bruch, } \\
\text { 2009, p. 390). }\end{array}$ \\
\hline $\begin{array}{l}\text { 15) Charisma } \\
\text { under crisis } \\
\text { revisited: } \\
\text { Presidential } \\
\text { leadership, } \\
\text { perceived leader } \\
\text { effectiveness, and } \\
\text { contextual } \\
\text { influences. }\end{array}$ & $\begin{array}{l}\text { 2012/ The Leadership } \\
\text { Quarterly }\end{array}$ & $\begin{array}{l}\text { Davis, K. M., \& } \\
\text { Ph.D. Candidate at the } \\
\text { Rawls College of } \\
\text { Business, Texas Tech } \\
\text { University } \\
\text { Gardner, W. L. } \\
\text { DBA, Florida State } \\
\text { University Professor in } \\
\text { Organizational Behavior } \\
\text { and Leadershipand Chair } \\
\text { Rawls College of } \\
\text { Business, Texas Tech } \\
\text { University }\end{array}$ & $\begin{array}{l}\text { - Empirical study } \\
\text { - Examines } \\
\text { "charismatic } \\
\text { rhetoric" used by } \\
\text { leaders under the } \\
\text { influence of crisis } \\
\text { (Davis \& Garder, } \\
\text { 2012, p. 921). } \\
\text { - Explores "leader } \\
\text { charismatic } \\
\text { rhetoric across two } \\
\text { major crises" (p. } \\
\text { 918). } \\
\text { - Utilizes "inductive } \\
\text { approach to theory } \\
\text { generation" (Davis }\end{array}$ & $\begin{array}{l}\text { - Adds to "the } \\
\text { scholarly debate } \\
\text { about the degree } \\
\text { to which crisis is } \\
\text { an antecedent for } \\
\text { charismatic } \\
\text { leadership by } \\
\text { considering the } \\
\text { patterns of leader } \\
\text { charismatic } \\
\text { rhetoric across } \\
\text { different crises" } \\
\text { (David \& } \\
\text { Gaardner, 2012, p. } \\
\text { 919). } \\
\text { Advances the }\end{array}$ \\
\hline
\end{tabular}




\begin{tabular}{|l|l|l|l|}
\hline & & $\begin{array}{l}\text { \& Gardner, 2012, } \\
\text { p. 918). }\end{array}$ & $\begin{array}{l}\text { literature by } \\
\text { providing "a } \\
\text { deeper }\end{array}$ \\
& & & $\begin{array}{l}\text { understanding of } \\
\text { the dynamic } \\
\text { relationship } \\
\end{array}$ \\
& & & $\begin{array}{l}\text { between crisis and } \\
\text { the emergence of } \\
\text { charismatic }\end{array}$ \\
& & & rhetorical \\
& & & leadership" (Davis \\
& & & Garder, 2012, \\
& & & p. 919). \\
\hline
\end{tabular}

According to Yukl (2010), sociologist Max Weber intensely influenced theories of charismatic leadership (p. 261). Yukl explained that according to Weber's theory, "charisma occurs during a social crisis, when a leader emerges with a radical vision that offers a solution to the crises and attracts followers who believe in the vision" (p. 261). Yukl also contended that, "in the past two decades, several social scientists formulate newer versions of the theory to describe charismatic leadership in organizations" (p. 261). Therefore, making this topic not only timely in the 21 st century, but also important.

Unquestionably, the literature reviews revealed that since the term "charisma" first appeared in the field of social sciences, the literature has advanced, and it presents a range of diverse studies, both theoretical and empirical, as well as interdisciplinary scholarly discussions. Over the years, scholars seemed to have attempted to advance the term charisma (e.g. Conger \& Kanungo, 1987), and re-examined it under new lenses (e.g. Fiol, Harris, \& House, 1999), to measure it (e.g. Levine, Muenchen, \& Brooks, 2010), to challenge its value (e.g. Antonakis, Fenley, \& Liechti, 2011) and to provide a greater understanding of charismatic leadership (Davis, \& Gardner, 2012).

Conger and Kanungo (1987) introduced a theory of charismatic leadership, contending that organizational scholars have greatly ignored charismatic leadership and focusing on, "the assumption that charisma is an attributional phenomenon" (p. 637). Meanwhile, Fiol et al. (1999) draw attention to process management, developing and testing a, "process model of charismatic leadership" (p. 449).

At the end of the first decade of the 21st century, Antonakis, Fenley and Liechti (2011) advanced the literature on charismatic leadership by suggesting that leaders could learn charisma, thus, "shedding light on the charisma phenomenon" (p. 390), and directly challenging Weber's (1947) suggestion of, "charisma as a gift of the body and spirit not accessible to everybody" (p. 375). 


\subsection{The Discussion on Charismatic Leadership in the 1980's and Early 1990's}

During the 1980's, scholars became exceptionally attracted by the, "emotional and symbolic aspects of leadership" (Yukl, 2010 p. 261). Some scholars, such as Conger and Kanungo (1987), suggested that organizational scholars have ignored charismatic leadership, and proposed an innovative "model linking organizational contexts to charismatic leadership.... [a] behavioral framework for studying charisma" (Conger \& Kanungo, 1987, p. 637- 639).

The Conger and Kanungo's (1987) model viewed charisma, "as a set of dispositional attributions by followers and as a set of leaders' manifest behaviors" (Conger \& Kanungo, 1987, p. 645). In spite of the fact that Conger and Kanungo (1987) indicated that Weber's (1947) conceptualization was limited as a result of an absence of "specificity", they agreed that charismatic authority is unpredictable (Conger \& Kanungo, 1987, p. 638- 644).

Nevertheless, the authors advanced the studies of charismatic leadership by presenting a model that, "builds in the idea that charisma is an attributional phenomenon" (Conger \& Kanungo, 1987, p. 639). Seeking to test empirically the theory, they suggested a creation of a questioner, "including the attributes believed to characterize charismatic leaders as well as those cited in the literature for other forms of leadership" (Conger \& Kanungo, 1987, p. 645).

In revisiting the theory, Shamir, House and Arthur (1993) introduced an argument linking charismatic and transformational leadership stating, "that charismatic leadership has its effects by strongly engaging followers' self-concepts in the interest of the mission articulated by the leader" (p. 577). Hence, Shamir and colleagues advanced charismatic leadership by offering, "a theoretical extension of current theories of charismatic and transformational leadership" (p. 590).

Seven years later, still contending that not enough empirical research was done concerning Weber's (1947) theory Conger and Kanungo (1994) addressed their own call for a "development of questionnaire measure of perceived behavioral dimensions of charismatic leadership proposed by [their] previous model (Conger \& Kanungo 1987, 1988)" (Conger \& Kanungo, 1994, p. 439). The Conger-Kanungo Scale of Charismatic Leadership emerged as a tool to advancement of the construct. To develop the scale, Conger and Kanungo collected data from 488 managers in four different organizations in North America, seeking, "to operationalize the charismatic leadership role of managers in organizations by developing reliable and valid questionnaire measures of the perceived behavioral attributes identified by the Conger-Kanungo model" (Conger \& Kanungo, 1994, p. 443). The result of the study was findings that supported the existence of behavioral dimensions in charismatic leadership (Conger \& Kanungo, 1994).

It is important to call attention that five years earlier, Howell and Frost (1989), addressing the call for empirical studies in the field of charismatic leadership, also added to the scholarly discussion, but without developing a questioner. The authors conducted a study with 144 undergraduates at the University of British Columbia, in which participants completed, "a task under the direction of either a charismatic, structuring, or considerate leader" (Howell \& Frost, 1989, p. 243). The results of the experiment indicated that, "individuals with 
charismatic leaders" presented "higher adjustment to their leader than individuals with considerate leaders" (Howell \& Frost, 1989, p. 263).

Howell and Frost (1989) greatly contributed to the theory of charismatic leadership, by validating that charisma, "can be studied under controlled laboratory conditions", and by suggesting that individuals "can be trained to exhibit charismatic behavior" (p. 265). Howell and Frost recommend numerous questions for progression of charismatic leadership, such as questions related to, "personality traits" and "situational determinants of charismatic leadership" (p. 266). Their paradigm suggests one of many approaches leading to becoming a charismatic leader regardless of prior leadership orientation.

Other scholars, such as Levine Muenchen and Brooks (2010), and most recently, Davis and Gardner (2012), later addressed empirically some of the questions posted by Howel and Frost (1989), related to "personality traits" and "situational determinants" (Howell \& Frost, 1989, p. 266).

\subsection{The Discussion on Charismatic leadership Late 1990's and 2000}

The end of the 20st century brought an intense and thought-provoking discussion among scholars regarding charismatic leadership. For example, Yukl (1999) started by challenging many aspects of charismatic leadership, including its broad value and by stating that, "the potential for using charismatic leadership to improve organizations seems limited, and it may be warranted only in special situations" (p. 301). Yukl's (1999) evaluation of charismatic and transformational leadership theories suggested that, "most of the theories have conceptual weaknesses", and that limit the successful explanation of "leadership phenomenon" (p. 285). Yukl went further and described how earlier theories presented weakness such as, "ambiguous constructs, insufficient description of explanatory processes, a narrow focus on dyadic processes, omission of some relevant behaviors, insufficient specification of limiting conditions (situational variables), and a bias toward heroic conceptions of leadership" (p. 286).

Furthermore, Yukl's (1999) conceptualization of charismatic and transformational leadership and, "the extent to which they are similar and compatible" (p. 298) caused mixed reactions in the scholarly community. Beyer (1999), a proponent of Weber's (1947) sociological approach, commented on Yukl's article, indicating that a departure from "Weber's original conception" could "blur the distinctiveness of the charismatic form of leadership by ignoring or downplaying integral aspects of charisma, especially the precipitating crisis, the radical vision, and subsequent systemic change" (p. 307). In the argument, Beyer explained that, "neo-charismatic and transformational leadership paradigms have tamed the original conception of charisma advanced by Weber (1947) and, in the process, diluted its richness and distinctiveness" (p. 308). Beyer, however, acknowledged during the scholarly discussion, that Weber's (1947) definition of charisma could be a, "rare phenomenon" (p. 313). Beyer further advanced the theory by proposing that scholars start shifting the form of how they study leadership theories (Beyer, 1999) pondering, "that it is possible that the phenomenon of leadership is too situation specific to yield the kinds of generalizations that researchers are seeking” (p. 325). 
Complementing the scholarly discussion, Fiol, Harris and House (1999) addressed Yukl's (1999) call for more research on process. They developed a "process model" of charismatic leadership utilizing theories of "social meaning" (Fiol et al., 1999, p. 449). The model clarified "why and how the charismatic leader/follower interaction can generate social change" by presenting an analysis of 42 speeches of 20th century U.S. presidents (Fiol et al., 1999, p. 449). The results of the study indicated that, "charismatic leaders employ consistent communication strategies for breaking down, moving, and re-aligning the norms of their followers" (p. 450). Fiol and colleagues seemed to advance charismatic leadership construct by arguing that while Weber (1947) and Conger and Kanungo (1987) suggested that charismatic leaders can effect change using "frame re-alignment", the authors' analysis implied the opposite, that in fact what happens is that followers, "will align around a new interpretive frame, they must reverse their previous relationship with the existing socially dominant values" (Fiol et al., 1999, p. 459). Ultimately, seeking to "redirect attention" Fiol and colleagues appeared to shed new light on the field of charismatic leadership in addressing psychological and relational processes.

In a similar vein as Yukl (1999), but with a more holistic view, Conger (1999) examined the evolution of charismatic and transformational leadership in organizations, seeking to address its past and future by examining the developments of the theories, and by considering "leader behaviors \& their effects" along with "follower dispositions \& dependency dynamics", "context", "institutionalization and success forces", as well as, "liabilities of charismatic and transformational leaders" (p. 145).

Conger (1999) started by primarily looking at, "background forces driving the growth of interest" not only Charismatic leadership but also in transformational leadership (p. 147). Building up on Weber's (1947) conception of charisma as "essentially an unstable force" (as cited in Conger, 1999, p. 168), the author stated that some important theorists presented theories similar to those of Weber (like Conger and Kanungo model) while other scholars presented important differences (e.g. Conger, 1999).

Conger (1999) advanced the charismatic leadership scholarly discussion during the late 1990 's by contending that, "Weber's notion that charisma must ultimately institutionalize itself may no longer be accurate" due to the globalization, new realities, and new times (p. 170). Lastly, Conger revealed how far research and theories advanced, nevertheless, the author emphasized the idea that there are still areas to explore (p. 168).

Giving continuity to the discussion during late 1990's, Hunt, Boal, and Dodge (1999) questioned the absence of substantial studies concerning the intersection of crisis and charisma, suggesting that, "given the pervasiveness of arguments in the charismatic literature, especially Weberian-based, concerning the importance of crisis" (p. 445). Thus, these scholars challenged Weber's (1947) definition of leader as “extraordinary" (Hunt et at.,1999, p. 443).

In addition to challenging Weber's (1947) definition, Hunt et al. (1999), provided support for hypotheses that there are two forms of charisma; "visionary \& crisis-responsive" (p. 425). Hunt et al. (1999) advanced charismatic leadership by testing 191 college undergraduates, 
with results advocating that "If leaders respond to crisis, they will be perceived to be as charismatic as visionary charismatic leaders. Crisis alone does not create charisma but crisis and response does" (p. 443).

Finally, Conger, Kanungo and Menon (2000) seemed to close the 20st century Weberian's scholarly discussion utilizing the Conger and Kanungo charismatic leadership scale and to conduct a study with 252 managers. The results revealed a "strong connection between follower reverence and charismatic leadership" (Conger et al., 2000, p. 747). The significant finding of the study demonstrated that, "followers of charismatic leaders develop a reverence for their leader and that this appears to be based most strongly upon their perceptions of the leader's sensitivity to the environment" (Conger et al., 2000, p.760). The study certainly opened the doors to a discussion of Weber's (1947) notion that "leaders use their expertize in demonstrating the inadequacy of the traditional technology, rules, and regulations of the status quo as a means of achieving the shared vision and uses ideas as foundation for own ideas" (Conger et al., 2000, p. 751), to advance the theory by proposing that "Charismatic leadership behavior will be positively related to the followers' trust in that manager" (Conger et al., 2000, p. 750). The authors finalized the study by presenting a much-needed suggestion in the field for future studies, suggesting that future research should look at "follower effects" and "follower's attributions" and "will need to include explicit measurement of followers' attributions about the leadership unique qualities and the leader's motivations" (Conger et al., 2000, p. 764).

\section{Charismatic Leadership in the 21st Century}

Certainly, since its inception in the field of social sciences in 1947, the charismatic leadership theory seems to capture the attention of several scholars and diverse fields. The review of the literature revealed that Weber's (1947) definition, theory and initial concept of "charismatic authority" witnessed substantial increase in scholarly writing and discussions in the beginning of the twenty first century (e.g. Antonakis, Fenley \& Liechti, 2011; Davis \& Gardner 2012, Levine, Muenchen, \& Brooks 2010; Paul, Costley, Howell, Dorfman \& Trafimow, 2001; Walter \& Bruch, 2009).

For example, Paul et al. (2001), empirical study of "The effects of leadership messages on followers' self-concept accessibilities" (p.1821), investigated 379 undergraduate college students enrolled in four courses. The study suggested that, "charismatic and integrative leadership messages from a leader resulted in higher follower collective self-concept accessibilities than did routinized messages" (Paul et al., 2001, p.1821). The study also shed light on "the importance of a charismatic leader, even when charismatic messages have become routinized" (Paul et al., 2001, p.1821). Paul and colleagues concurred with Weber's (1947) notion of "charismatic and integrative leadership messages delivered by a leader will have greater effects than will routinized charismatic messages" (p. 1828). Additionally, Paul's et al., study highlighted the significance of charismatic leaders (p.1836), providing, "some empirical support for the views of Weber (1947)" and suggesting "that a leader remains important to the effectiveness of charismatic leadership even when charismatic messages have become routinized" (p. 1837). 
The 21st century also brought a noteworthy development in the field of leadership as it relates to charismatic leadership. For instance, Walter and Bruch's (2009) integration of previous literature into a comprehensive overview of charismatic leadership provided a comprehensive review of charismatic leadership behavior emergence looking to develop a "comprehensive perspective by integrating previous work into an overall conceptual framework" (Walter \& Bruch, 2009, p. 1428). The study questioned Weber's (1947) notion that crises are, "prerequisite for charismatic leadership" (Walter \& Bruch, 2009, p. 1433) (Walter \& Bruch, 2009, p. 1433). Walter and Bruch (2009) advanced the theory of charismatic leadership by suggesting that scholars such as Conger (1999) and Yukl (1999) have "neglected the origins of charismatic leadership behavior" (p. 1444). Lastly, Walter and Bruch (2009) outlined suggestions for potential future research such as the development of an empirical study about possible important factors "underrepresented" in the literature suggesting that future research should explore the inclusion of studies that could possibly advance the study of 'how leaders' immediate social context and larger work environment influence the 'dark', personalized aspects of their charismatic behavior" (Walter \& Bruch, 2009, p. 1444).

Levine, Muenchen, and Brooks (2010) added significantly to Yukl's (1999) and Byer's (1999) discussions about the intersection of charismatic and transformational leadership. Levine et al. conducted a study with 422 participants, noting that the measurement scales utilized to measure charismatic and transformational theories, "fail to adequately address and measure the communication behaviors that are believed to be a part of charisma" (p. 576). The study considered important scholars such as Weber (1947) and Conger (1989), and explained that the verbs and descriptors utilized more frequently to define charismatic leadership are: behavior, presence in a crisis; determination; communication of ideas; and communication of expectations (Levine et al., 2010, p. 580). The results of the study suggested that in order to comprehend the interception of transformational and charismatic leadership, scholars should develop, "a new leadership measurement that includes charismatic communication behaviors theories" (Levine et al., 2010, p. 576). Levine et al. expanded the literature by challenging current scales, arguing that they are not accurate, and by suggesting the creation of a leadership measurement instrument including, "charismatic communication behaviors" ( $p$. 589). Ultimately, Levine et al. offered direction for future research, including the incorporation of the "dimensions of charisma and charismatic behavior" and development of, "new measurement scales used in future research" (p. 589).

The 21 st century also presented another important study. Antonakis et al. (2011) addressed Weber's (1947) suggestion of charisma as a gift, "not accessible to everybody" (p. 375), and investigates possibilities to "teach individuals to behave more charismatically, and if changes in charisma affect leader outcomes" (p. 374). Antonakis' et al., experiment suggested that, "leaders appear charismatic because they use a wide array of verbal and nonverbal CLTs" "Charismatic Leadership Tactics" (p. 390). The study advanced the literature by, "shedding light on the charisma phenomenon" (Antonakis et al., 2011, p. 390). The study, "main contribution is that leadership is learnable" (Antonakis et al., 2011, p. 390).

Furthermore, addressing issues raised by Howell and Frost (1989) at the end of the 20st 
century, Davis and Gardner (2012) addressed situational determinants. Davis and Gardner, contribute to the discussion by adding to "the scholarly debate about the degree to which crisis is an antecedent for charismatic leadership by considering the patterns of leader charismatic rhetoric across different crises" (p. 919).

Davis and Gardner's (2012), computerized content analysis study investigated President George W. Bush's speeches and radio addresses following two different major crises. The investigation explored, "leader charismatic rhetoric across two major crises, longitudinally exploring potential long-term influences of charismatic rhetoric on perceptions of leader effectiveness" (Davis \& Gardner 2012, p. 918).

Davis and Gardner (2012) utilized an inductive approach in order to generate a theory of charismatic rhetoric constructs, and to "advance propositions regarding potential time and ceiling effects of charismatic rhetorical leadership on followers" (p. 918). With a textual approach, the authors stated that both Obama and Bush saw a decline in approval rating, "despite high levels of charismatic rhetoric", a pattern that is consistent with Weber's (1947) and other scholars "assertion that while charismatic leadership may be effective in eliciting initial follower support for the leader's vision, such support will not be sustained without tangible evidence of success in achieving that vision" (p. 928).

Davis and Gardner (2012) notably contributed to the literature of Charismatic Leadership by adding to, "the scholarly debate about the degree to which crisis is an antecedent for charismatic leadership by considering the patterns of leader charismatic rhetoric across different crises" (p. 919). The authors advanced the literature by providing "a deeper understanding of the dynamic relationship between crisis and the emergence of charismatic rhetorical leadership" (Davis \& Gardner, 2012, p. 919). Lastly, by shedding light to "potential follower effects of charismatic rhetoric in the form of perceptions of leader effectiveness" the authors seem to contribute to the advancement of literature (Davis \& Gardner, 2012, p. 919).

Ultimately, the 21 st century seemed to advance the literature slowly, but steadily, providing new directions and an emergence of new methods of studying the phenomenon, such as "computerized content analysis" (Davis and Gardner, 2102, p. 921). Suggestions of future research are also prominent, such as the necessity of scholars to, "focus on uncovering which learning processes are most relevant and how they can be managed and accelerated" (Antonakis et al., 2011, p. 390). Conversely, presentation of limitations and possibilities for future research are suggested such as, "future researchers should gather more complete data" (Antonakis et al., 2011, p. 391).

\section{Conclusion}

In this literature review the charismatic leadership construct was reviewed from its conception to the first decade of 21st century, by examining and summarizing different theoretical and empirical studies that highlighted various stages that the charismatic leadership theory has passed through, and its recent developments and methods of study.

The theory started with a sociological and physiological approach, and throughout the years, as scholars engaged in discussions, more dimensions and methods of study were added to the 
investigation of the theory. Scholars such as Beyer (1999) endorsed that the original theory succeeded in fulfilling its theoretical promises. Other scholars such as Conger and Kanungo (1987) criticized Weber's (1947) original construct of charismatic authority.

The study of the construct of charismatic theory is certainly very thought provoking, and its applications in educational organizational studies are boundless. The possibility of testing and understanding the charismatic leadership in high-pressure contexts is much needed for the advancement of the construct. A study of charismatic rhetoric of professors in diverse online and hybrid environments for doctoral studies appears to be numerous. For instance, future studies should seek to examine the extent of the use of charismatic rhetoric in online courses in highly demanding courses, such as doctoral courses, and its impact if any, on students' achievement.

Additionally, future studies should assess the significance, effects, and educational value, of charismatic rhetoric in doctoral programs. Ultimately, the literature search, reviews and construct show that no studies have addressed this topic thus far.

\section{References}

Antonakis, J., Fenley, M., \& Liechti, S. (2011). Can charisma be taught? Tests of two interventions.Academy of Management Learning \& Education, 10(3), 374-396. https://doi.org/10.5465/amle.2010.0012

Beyer, J. M. (1999). Taming and promoting charisma. The Leadership Quarterly, 10(2), 307-330. https://doi.org/10.1016/S1048-9843(99)00019-3

Conger, J. A., \& Kanungo, R. N. (1998). Charismatic leadership in organizations. Thousand Oaks, CA: Sage.

Conger, J. A. (1999). Charismatic and transformational leadership in organizations: an insider's perspective on these developing streams of research. The Leadership Quarterly, 10(2), 145-179. https://doi.org/10.1016/S1048-9843(99)00012-0

Conger, J. A., \& Kanungo, R. N. (1987). Toward a behavioral theory of charismatic leadership in organizational settings. The Academy of Management Review, 12(4), 637-647. https://doi.org/10.5465/amr.1987.4306715

Conger, J. A., \& Kanungo, R. N. (1994). Charismatic leadership in organizations: perceived behavioral attributes and their measurement. Journal of Organizational Behavior, 15(5), 439-452. https://doi.org/10.1002/job.4030150508

Conger, J. A., Kanungo, R. N., \& Menon, S. T. (2000). Charismatic leadership and follower $\begin{array}{llll}\text { effects. Journal of } & \text { Organizational Behavior, 21(7), }\end{array}$ https://doi.org/10.1002/1099-1379(200011)21:7<747::AID-JOB46>3.0.CO;2-J

Davis, K. M., \& Gardner, W. L. (2012). Charisma under crisis revisited: Presidential leadership, perceived leader effectiveness, and contextual influences. The Leadership Quarterly,23(1),918-933. https://doi.org/10.1016/j.leaqua.2012.06.001 
Fiol, C., Harris, D., \& House, R. (1999). Charismatic leadership: strategies for effecting social change. The Leadership Quarterly, 10(3), 449-482. https://doi.org/10.1016/S1048-9843(99)00021-1

Howell, J. M., \& Frost, P. J. (1989). A laboratory study of charismatic leadership. Organizational Behavior and Human Decision Processes, 43(2), 243-269. https://doi.org/10.1016/0749-5978(89)90052-6

Hunt, J. G., Boal, K. B., \& Dodge, G. E. (1999). The effects of visionary and crisis-responsive charisma on followers: an experimental examination of two kinds of charismatic leadership. The Leadership Quarterly, 10(3), 423-448.https://doi.org/10.1016/S1048-9843(99)00027-2

Levine, K. J., Muenchen, R. A., \& Brooks, A. M. (2010). Measuring transformational and charismatic leadership: why isn't charisma measured. Communication Monographs, 77(4), 576-591. https://doi.org/10.1080/03637751.2010.499368

Paul, J., Costley, D. L., Howell, J. P., Dorfman, P. W., \& Trafimow, D. (2001). The effects of charismatic leadership on followers' self-concept accessibility. Journal of Applied Social Psychology, 31(9), 1821-1844. https://doi.org/10.1111/j.1559-1816.2001.tb00206.x

Shamir, B., House, R. J., \& Arthur, M. B. (1993). The motivational effects of charismatic leadership: a self-concept based theory. Organization Science, 4(4), 577-594. https://doi.org/10.1287/orsc.4.4.577

Walter, F., \& Bruch, H. (2009). An affective events model of charismatic leadership behavior: a review, theoretical integration, and research agenda. Journal of Management, 35(6), 1428-1452. https://doi.org/10.1177/0149206309342468

Weber, M. (1947). The theory of social and economic organization. Free Press.

Yukl, G. (1999). An evaluation of conceptual weaknesses in transformational and charismatic leadership theories. The Leadership Quarterly, 10(2), 285-305. https://doi.org/10.1016/S1048-9843(99)00013-2

Yukl, G. (2010). Leadership in organizations. (7th ed.). Prentice Hall.

\section{Copyright Disclaimer}

Copyright for this article is retained by the author(s), with first publication rights granted to the journal.

This is an open-access article distributed under the terms and conditions of the Creative Commons Attribution license (http://creativecommons.org/licenses/by/3.0/). 